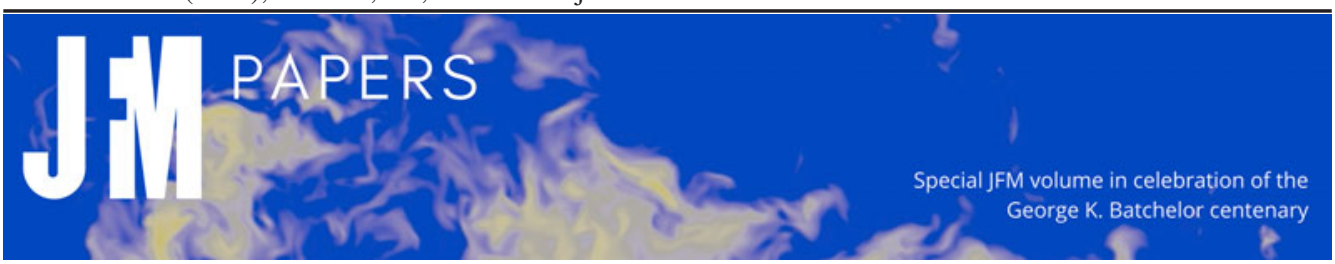

\title{
On the nature of intermittency in a turbulent von Kármán flow
}

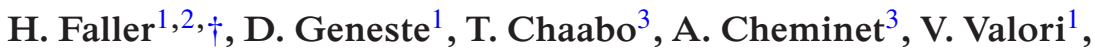 \\ Y. Ostovan ${ }^{3}$, L. Cappanera ${ }^{4}$, Ch. Cuvier ${ }^{3}$, F. Daviaud ${ }^{1}$, J.-M. Foucaut ${ }^{3}$, \\ J.-L. Guermond ${ }^{5}$, J.-Ph. Laval ${ }^{3}$, C. Nore ${ }^{2}$, V. Padilla ${ }^{1}$, C. Wiertel ${ }^{1}$ and \\ B. Dubrulle ${ }^{1}$ \\ ${ }^{1}$ Université Paris-Saclay, CEA, CNRS, SPEC, CEA Saclay, 91191 Gif sur Yvette CEDEX, France \\ ${ }^{2}$ Laboratoire d'Informatique pour la Mécanique et les Sciences de l'Ingénieur, Université Paris-Saclay, \\ CNRS, LIMSI, 91400 Orsay, France \\ ${ }^{3}$ Univ. Lille, CNRS, ONERA, Arts et Metiers Institute of Technology, Centrale Lille, UMR \\ 9014-LMFL-Laboratoire de Mécanique des Fluides de Lille-Kampé de Fériet, F-59000 Lille, France \\ ${ }^{4}$ Department of Mathematics, University of Houston, Houston, TX 77204-3008, USA \\ ${ }^{5}$ Department of Mathematics, Texas A\&M University 3368 TAMU, College Station, \\ TX 77843-3368, USA
}

(Received 13 May 2020; revised 16 September 2020; accepted 15 October 2020)

We have conducted an extensive study of the scaling properties of small scale turbulence using both numerical and experimental data of a flow in the same von Kármán geometry. We have computed the wavelet structure functions, and the structure functions of the vortical part of the flow and of the local energy transfers. We find that the latter obey a generalized extended scaling, similar to that already observed for the wavelet structure functions. We compute the multi-fractal spectra of all the structure functions and show that they all coincide with each other, providing a local refined hypothesis. We find that both areas of strong vorticity and strong local energy transfer are highly intermittent and are correlated. For most cases, the location of local maximum of the energy transfer is shifted with respect to the location of local maximum of the vorticity. We, however, observe a much stronger correlation between vorticity and local energy transfer in the shear layer, that may be an indication of a self-similar quasi-singular structure that may dominate the scaling properties of large order structure functions.

Key words: intermittency, turbulence theory, fractals

$†$ Email address for correspondence: hugues.faller@ normalesup.org A video presentation about this research work can be found at: https://www.cambridge.org/dubrulle-abstract. 


\section{H. Faller and others}

\section{Introduction}

In 1949, Batchelor \& Townsend (1949) published the first experimental account about inconsistencies in the original turbulence theory of Kolmogorov (1941). Measuring with a hot wire, the streamwise velocity $u$ gives access to the velocity derivatives along the flow direction $x$. They observed that various flatnesses of the distribution of velocity derivatives,

$$
\left.\alpha(n)=\overline{\left(\frac{\partial^{n} u}{\partial x^{n}}\right)^{4}} / \overline{\left[\left(\frac{\partial^{n} u}{\partial x^{n}}\right)^{2}\right.}\right]^{2},
$$

increase both with $n$ and with the Reynolds number $R e$. Their interpretation was that $\partial^{n} u / \partial x^{n}$ fluctuates in a manner that is more markedly intermittent as $n$ or $R e$ increases, a fact confirmed by oscillograms of the velocity field derivatives. The finding was summarized in a remarkable concise but visionary paragraph stating that 'energy associated with large wave-number is very unevenly distributed in space. There appear to be isolated regions in which the large wave-numbers are "activated", separated by regions of comparative quiescence. This spatial inhomogeneity becomes more marked with increase in the order of the velocity derivative, i.e. with increase in the wave-number. It is suggested that the spatial inhomogeneity is produced early in the history of the turbulence by an intrinsic instability, in the way that a vortex sheet quickly rolls up into a number of strong discrete vortices. Thereafter the inhomogeneity is maintained by the action of the energy transfer'. As noted by Moffatt (2002), such a finding describes precisely the now well-admitted intermittency of the vorticity field. At the same time, it provides a clear scenario of the building of intermittency and inhomogeneity via the first Kelvin-Helmholtz instability (roll up of the vorticity sheet), then breaking into discrete blobs of vorticity and finally action of the energy transfer, that allows maintenance of the resulting inhomogeneity along scales.

The result of Batchelor and Townsend only concerns intermittency at the dissipative scales. The Kolmogorov (1962) refined theory allows us to connect intermittency of the local energy dissipation with (intermittent) correction to scaling of the energy spectrum, or of the velocity structure functions up to the inertial scales. In this picture, there is a direct link between the 'active' regions of intense local dissipation, and the intermittent corrections to scaling. Because the areas of intense dissipation are observed to arise in the vicinity of vorticity filaments (Vincent \& Meneguzzi 1994), or sheets (Moisy \& Jiménez 2004), there have been several attempts to link intermittency exponents and vorticity-based coherent structures, with sometimes conflicting conclusions. By computing wavelet-based velocity and vorticity structure functions, Kestener \& Arneodo (2004) found the same intermittency in a $256^{3}$ numerical simulation at $R_{\lambda}=140$.

However, Nguyen et al. (2019) found a different multi-fractal spectrum between the two fields in a numerical simulation at the same $R_{\lambda}$ but with a large resolution $768^{3}$ (see figures 7 and 8 in Nguyen et al. (2019)). From the experimental side, Paret \& Tabeling (1998) used a simultaneous monitoring of local pressure and velocity in an experimental flow at large Reynolds number to find that the intermittency is decreased when removing from the velocity signal the portions corresponding to very low pressure events (presumably tracing vortex cores). The procedure was improved using wavelet filtering by Chainais, Abry \& Pinton (1999), to conclude that the coherent structures do affect the intermittency by acting on the way the cascade develops. Altogether, these results suggest that the vorticity is not the only important ingredient of the intermittency, and that energy transfers should be somehow taken into account, as first argued by Kraichnan (1975). 
Indeed, a direct link between the intermittent exponent and instantaneous partial local energy transfer at the Kolmogorov scale was found in an experimental turbulent swirling flow using conditional statistics (Debue et al. 2018; Dubrulle 2019). At this time, only velocity measurements on a plane were available, meaning that a fraction of the local energy transfer was missing, and that the vorticity field could not be computed, preventing investigation of possible correlations between them and with intermittent corrections. Thanks to an outstanding experimental and numerical effort, we now have at our disposal both three-dimensional (3-D) time and space resolved velocity measurements and numerical data in the same geometry (Debue et al. 2020; Cappanera et al. 2021). The goal of the present paper is thus to gather all results, and investigate how much local energy transfers and vorticity are correlated, and correlated with intermittent corrections to scaling.

\section{Definitions}

Here, we define the basic tools that we use in our analysis. The time dependence of the velocity field has been removed in the notations for convenience.

\subsection{Vorticity}

The vorticity is a well-known quantity in turbulence. Its magnitude will be mostly used here as

$$
\omega=\|\nabla \times u\|
$$

\subsection{Increments and structure functions}

The traditional basic tool to study intermittency is via scaling of the velocity structure functions of order $p$, defined as

$$
S_{p}(\ell)=\left\langle\left(\delta_{\ell} u\right)^{p}\right\rangle_{\|\ell\|=\ell} .
$$

Here, $\delta_{\ell} u$ is the longitudinal velocity increment at scale $\ell$, defined as

$$
\delta_{\ell} u=\hat{\ell} \cdot(\boldsymbol{u}(\boldsymbol{x}+\boldsymbol{\ell})-\boldsymbol{u}(\boldsymbol{x})),
$$

where $\hat{\ell}$ is the increment direction. Throughout the paper, the notation $h$ refers to the local Hölder regularity of $\delta_{\ell} u$ so that $\delta_{\ell} u \sim \ell^{h}$. Muzy, Bacry \& Arneodo (1991) argued that wavelet-based velocity increments $\delta W_{\ell}$ may provide more robust results, via the so-called wavelet transform modulus maxima method. The use of wavelets (Farge 1992; Schneider \& Vasilyev 2010) allows us to separate strong vorticity structures from the noise. We use a wavelet such that the first moment vanishes. This allows us to explore Hölder exponents lower than one. The velocity increments are defined via the wavelet transform of the tensor $\partial_{j} u_{i}$

$$
G_{i, j}(\boldsymbol{x}, \ell)=\int \nabla_{j} \phi_{\ell}(\boldsymbol{r}) u_{i}(\boldsymbol{x}+\boldsymbol{r}) \mathrm{d} \boldsymbol{r},
$$

where $\phi_{\ell}(x)=\ell^{-3} \phi(x / \ell)$ is a smooth non-negative function with unit integral. From this, we get the wavelet velocity increments as

$$
\delta W_{\ell}(\boldsymbol{x})=\ell \max _{i, j}\left|G_{i, j}(\boldsymbol{x}, \ell)\right| .
$$

The interest of such a formulation is that it allows us to define a velocity increment connected with the scaling properties of the vorticity, by considering the wavelet velocity 


\section{H. Faller and others}

increment built from the anti-symmetric part of the tensor $\partial_{j} u_{i}$, so that

$$
\delta \Omega_{\ell}(\boldsymbol{x})=\frac{\ell}{2} \max _{i, j}\left|G_{i, j}-G_{j, i}\right|
$$

\subsection{Local energy transfer}

As argued by Kraichnan (1975) and Meneveau (1991), the local energy transfer at scale $\ell$ may be important to understanding the origin of intermittency. For example, consider the local refined hypothesis of Kolmogorov (1962) stating that $\left(\delta u_{\ell}\right)^{3} \sim \epsilon_{\ell} \ell$, where $\sim$ means 'has the same statistical properties' and $\epsilon_{\ell}$ is the energy dissipation over a ball of scale $\ell$. It seems more logical in such a formula to replace $\epsilon_{\ell}$ by the local energy transfer at scale $\ell$. This is the local refined hypothesis of Kraichnan. The latter can be computed as

$$
\mathscr{D}_{\ell}^{I}=\frac{1}{4} \int \nabla \phi_{\ell}(\xi) \cdot \delta_{\xi} u\left(\delta_{\xi} u\right)^{2} \mathrm{~d} \boldsymbol{\xi}
$$

where $\phi_{\ell}$ is a smooth non-negative function with unit integral. In the following, we choose the same function for computing $\delta W_{\ell}, \delta \Omega_{\ell}$ and $\mathscr{D}_{\ell}^{I}$, and take it as a Gaussian function $\phi_{\ell}(\boldsymbol{x})=\left(1 /(\ell \sqrt{2 \pi})^{3}\right) \exp \left(-\|x\|^{2} / 2 \ell^{2}\right)$.

As discussed in Dubrulle (2019), these local energy transfers compete at each scale with the local energy dissipation defined as

$$
\mathscr{D}_{\ell}^{v}=\frac{v}{2} \int \nabla^{2} \phi_{\ell}(\xi)\left(\delta_{\xi} u\right)^{2} \mathrm{~d} \xi .
$$

The local refined hypothesis of Kraichnan (1975) can then be expressed in a more general fashion following Dubrulle (1994). Replacing $\ell$ by $\left\langle\delta W_{\ell}^{3}\right\rangle / \epsilon$ and $\epsilon_{\ell}$ by $\left\langle\mathscr{D}_{\ell}^{I}\right\rangle$ leads to an extended self-similarity as proposed by Dubrulle (2019):

$$
\frac{\left\langle\delta W_{\ell}^{p}\right\rangle}{\left\langle\delta W_{\ell}^{3}\right\rangle^{p / 3}} \propto \frac{\left\langle\left|\mathscr{D}_{\ell}^{I}\right|^{p / 3}\right\rangle}{\left\langle\left|\mathscr{D}_{\ell}^{I}\right|\right\rangle^{p / 3}} .
$$

\subsection{Scaling exponents}

The scaling exponents of the velocity structure functions $\zeta(p)$ are defined so that $S_{p}(\ell) \sim$ $\ell^{\zeta(p)}$ in the inertial range. The latter is defined in the range of scales where the Kolmogorov 4/5th law applies, namely

$$
S_{3}(\ell)=-\frac{4}{5} \epsilon \ell,
$$

where $\epsilon$ is the energy dissipation rate per unit mass. By definition, we thus have $\zeta(3)=1$. Intermittency corrections are thus encoded by $\tau(p)=\zeta(p)-p / 3$, with $\tau(3)=0$. By extension, we define the scaling exponents of $\delta W_{\ell}, \delta \Omega_{\ell}$ and $\mathscr{D}_{\ell}^{I}$ via the compensated 
structure functions as

$$
\left.\begin{array}{c}
\tilde{S}_{W}(p)=\frac{\left\langle\delta W_{\ell}^{p}\right\rangle}{\left\langle\delta W_{\ell}^{3}\right\rangle^{p / 3}} \propto \ell^{\tau_{W}(p)}, \\
\tilde{S}_{\Omega}(p)=\frac{\left\langle\delta \Omega_{\ell}^{p}\right\rangle}{\left\langle\delta \Omega_{\ell}^{3}\right\rangle^{p / 3}} \propto \ell^{\tau_{\Omega}(p)}, \\
\tilde{S}_{D}(p)=\frac{\left\langle\left|\mathscr{D}_{\ell}^{I}\right|^{p}\right\rangle}{\left\langle\left|\mathscr{D}_{\ell}^{I}\right|\right\rangle^{p}} \propto \ell^{\tau_{D}(p)},
\end{array}\right\}
$$

in the inertial range of scales. Note that, in the following, we discriminate between the structure functions and their compensated version by a tilde. By definition $\tau_{W}(3)=$ $\tau_{\Omega}(3)=\tau_{D}(1)=0$. Note that the refined similarity hypothesis given by (2.9) states that $\tau_{W}(p)=\tau_{D}(p / 3)$.

\subsection{Multi-fractal spectrum}

The multi-fractal spectrum is defined as the Legendre transform of the scaling exponents of the velocity structure functions $\zeta(p)$, so that

$$
C(h)=\max _{p}(-p h+\zeta(p)) .
$$

Here, we adopt the language of large deviations, where $C(h)$ is the rate function of the local scaling exponent. It corresponds to $3-D(h)$ where $D(h)$ is the dimension of the set of points with local exponent $h$ in the Frisch \& Parisi (1985) interpretation. By extension, we also define the multi-fractal spectra of $\delta W_{\ell}, \delta \Omega_{\ell}$ and $\mathscr{D}_{\ell}^{I}$ as

$$
\left.\begin{array}{l}
C_{W}(h)=\max _{p}\left(-p h+\tau_{W}(p)\right), \\
C_{\Omega}(h)=\max _{p}\left(-p h+\tau_{\Omega}(p)\right), \\
C_{D}(h)=\max _{p}\left(-p h+\tau_{D}(p)\right) .
\end{array}\right\}
$$

Due to properties of the Legendre transform, $C_{W}$ can be directly compared with $C(h)$ provided a shift

$$
C_{W}\left(h-\frac{\zeta(3)}{3}\right)=C(h) .
$$

Moreover, if the refined similarity hypothesis (2.9) is satisfied, then $C_{W}(h)=C_{D}(3 h)$.

\section{Description of the von Kármán datasets}

The framework of our investigation is turbulence in the von Kármán geometry. The fluid of viscosity $v$ is confined in a cylinder of radius $R=10 \mathrm{~cm}$ and height $H=1.8 R$, and set into motion by two counter-rotating impellers, rotating at the same frequency $F$. It is well known that the von Kármán flow is globally inhomogeneous and anisotropic. Most of the measurements are performed in the centre of the tank, where the flow is more homogeneous and isotropic (Ouellette et al. 2006). In the following, we use $R$ and $1 / 2 \pi F$ as units of length and time respectively, so that the global Reynolds number of the flow is $R e=2 \pi F R^{2} / \nu$. The properties of the turbulence, such as global dissipation 


\section{H. Faller and others}

$\begin{array}{lccccccccc}\text { Case } & F(\mathrm{~Hz}) & \text { Grid points } & R e & R_{\lambda} & \epsilon(\operatorname{adim}) & T_{\text {int }} & \eta(\mathrm{mm}) & \Delta x(\mathrm{~mm}) & \text { Symbol } \\ \text { A } & 5 & 89 \times 65 & 3.1 \times 10^{5} & 610 & 0.045 & 2.00 & 0.016 & 2.1 & \bullet \\ \mathrm{B} & 5 & 77 \times 79 & 3.1 \times 10^{5} & 920 & 0.045 & 3.02 & 0.016 & 0.49 & \mathbf{\square} \\ \mathrm{C} & 5 & 162 \times 157 & 3.1 \times 10^{5} & 890 & 0.045 & 2.92 & 0.016 & 0.24 & \checkmark \\ \mathrm{D} & 1 & 77 \times 80 & 4.1 \times 10^{4} & 300 & 0.045 & 2.71 & 0.073 & 0.49 & \mathbf{\Delta} \\ \mathrm{E} & 1.2 & 151 \times 174 & 5.8 \times 10^{3} & 72 & 0.045 & 1.73 & 0.32 & 0.24 & \star \\ \text { T-1 } & 5 & 149 \times 103 \times 20 & 3.1 \times 10^{5} & 890 & 0.045 & 2.92 & 0.016 & 0.35 & \circ \\ \text { T-2 } & 1 & 139 \times 101 \times 20 & 6.3 \times 10^{4} & 390 & 0.045 & 2.84 & 0.054 & 0.35 & \square \\ \text { T-3 } & 0.5 & 148 \times 103 \times 20 & 3.1 \times 10^{4} & 250 & 0.045 & 2.59 & 0.09 & 0.35 & \diamond \\ \text { T-4 } & 0.1 & 149 \times 100 \times 20 & 6.3 \times 10^{3} & 80 & 0.045 & 1.84 & 0.3 & 0.35 & \triangle \\ \text { DNS } & \frac{1}{2 \pi} & 400 \times 800 \times 509 & 6 \times 10^{3} & 72 & 0.045 & 1.70 & 0.37 & 0.1-0.4 & -\circ-\end{array}$

Table 1. Parameters describing the main datasets used in this paper. Here, $F$ is the rotation frequency of the impellers in $\mathrm{Hz} ; R e$ is the Reynolds number based on $F$ and the radius of the tank; $R_{\lambda}$ is the Taylor-microscale Reynolds number; $\epsilon$ is the global dimensionless energy dissipation; $T_{\text {int }}$ is the integral time scale; $\eta$ is the Kolmogorov dissipation length scale; and $\Delta x$ represents the spatial resolution in the measurements and the DNS. The last column shows the symbols used to represent the experimental datasets. SPIV stands for stereoscopic particle image velocimetry (three component measurements of the velocity on a plane), while TPIV is tomographic particle image velocimetry (three component measurements of the velocity in a cuboid). Since the DNS is dimensionless (the cylinder radius is one), we express $\Delta x$ and $F$ in terms of the experimental cylinder radius $(R=10 \mathrm{~cm})$ and DNS advection time scale $(T=1)$ for a better comparison.

and root-mean-square velocity, depend on the shape of the propeller. In the following, we work with the so-called scooping TM87 propeller, described and discussed at length in Debue (2019), Cappanera et al. (2021) and Dubrulle (2019). In this case, the flow is fully turbulent for $R e \geq 6000$ and the non-dimensional dissipation per unit mass is $\epsilon=0.045$ in the turbulent regime. We define an integral time scale $T_{i n t}=(1 / 2)\left(\boldsymbol{u}_{r m s}^{2} / \epsilon\right)$ expressed in units of the advection time. It is of order one for all the datasets. The Kolmogorov length and time are respectively $3 \times 10^{-4} \mathrm{~m}$ and $4 \times 10^{-2} \mathrm{~s}$ at $R e=6000$, which are accessible to both modern particle velocity measurements and direct numerical simulations on a supercomputer. This allows us to combine both numerics and experiments to explore the nature of intermittency in such a turbulent flow.

\subsection{Experimental datasets}

Experimental data were collected with particle image velocimetry (PIV) techniques. Hereafter, data referred to by the letters A-E refer to stereoscopic PIV (SPIV) where the three components (3C) are deduced by two-dimensional (2-D) measurements on a plane using two cameras (2-D-3C). This dataset is described in Dubrulle (2019), and corresponds either to global velocity measurement in the whole tank (set A) or data taken in a small window zoomed in the centre region of the tank (cases B-E). In each case, either the frequency of the impellers or the viscosity of the fluid were tuned to modify the Reynolds number and the Kolmogorov scale. The corresponding parameters for each case are summarized in table 1 . All the SPIV data were acquired at $15 \mathrm{~Hz}$, resulting in time uncorrelation between two successive measurements.

Corresponding spatial resolution can be found in table 1 . Data named by T-1 to T-4 refer to tomographic PIV (TPIV) where the three components are deduced by tomographic reconstruction (multiplicative algebraic reconstruction technique (MART) method) in a volume using five cameras (three dimensions, three components: 3-D-3C). This whole 


\section{On the nature of intermittency in a von Kármán flow}

dataset is acquired at the centre of the tank in water by Ostovan et al. (2019) and described in Debue (2019). Velocity fields corresponding to different viscosity or different impeller frequency were measured, as summarized in table 1 . In all experimental cases, time uncorrelation between two measurements is observed. Tomographic reconstruction was done with 5 MART iterations keeping low value of the ghost ratio, less than $10 \%$; $\epsilon$ and $\eta$ are computed using global dissipation measured by torquemeters while $R_{\lambda}$ is deduced from PIV data. The measurement region and PIV spatial resolution $\Delta x$ have an impact on the root-mean-square velocity and Taylor microscale defining $R_{\lambda}$, therefore leading to different values of $R_{\lambda}$ for a given viscosity $v$ and frequency $F$. As expressed in table 1 , the experiment grid size is rather small, but a very large number of snapshots, of the order of $10^{4}$, are available for each dataset. A statistical convergence study for experimental quantities is presented in the appendix of Debue et al. (2018).

\subsection{Numerical dataset}

The numerical simulations are performed using the SFEMaNS code (spectral/finite element code for Maxwell and Navier-Stokes equations). This code uses a hybrid spatial discretization combining spectral and finite elements. In a nutshell, the approximation in space is done by using a Fourier decomposition in the azimuthal direction and the continuous Hood-Taylor Lagrange finite element for $(r, z)$ dependencies. The moving counter-rotating impellers are accounted for by using a pseudo-penalty technique. The performance of this technique is discussed in detail in $\$ 2.4$ of Nore et al. (2018) where shorter impellers are used. Visualizations of turbulent structures, global quantities (such as kinetic energy or fluctuations level) and spatial spectra obtained from hydrodynamical simulations are in agreement with experimental observations (see $\S 3$, Nore et al. (2018)) and thus validate the numerical method.

The rotation frequency is set to $1 / 2 \pi$ and the viscosity is chosen so that the global Reynolds number is $6 \times 10^{3}$. We use 250 complex modes for the Fourier decomposition and the spatial resolution is between $1.3 \times 10^{-3} R \simeq \eta / 3$ and $4 \times 10^{-3} R \simeq \eta$. This unstructured, irregular, mesh is refined in the middle of the tank, where the strongest turbulence takes place, with no preferred direction for the mesh. Contrary to the experimental set-up, which requires a cooling system, the numerical simulations set the impellers external disks in contact with the top and bottom lids of the tank.

The Reynolds number of the direct numerical simulations (DNS) may appear rather low. It was chosen so as to match the lower Reynolds number $R e=6 \times 10^{3}$ of the experiments. This choice of Reynolds number was originally made in the experiments because $6 \times 10^{3}$ is the lowest Reynolds number at which we know that the flow is fully turbulent, since it is the beginning of the plateau of the dissipation anomaly (see discussion in figure 14 of Dubrulle (2019)). On the other hand, it is the flow with the largest Kolmogorov scale, enabling us to match the highest spatial resolution we could achieve in our experimental PIV. But once more, we stress that it corresponds to a fully turbulent flow. The analysed dataset has 21 uncorrelated snapshots. The high number of grid points $3 \times 10^{8}$ counter-balances a low number of snapshots. A convergence study of computed statistical quantities is presented in the appendix A.

\section{Comparison between local energy transfer and vorticity}

\subsection{Fields}

Figure 1 compares typical instantaneous experimental snapshots of the vorticity amplitude with the local energy transfer. This figure corresponds to a slice of an experimental 


\section{H. Faller and others}

(a)

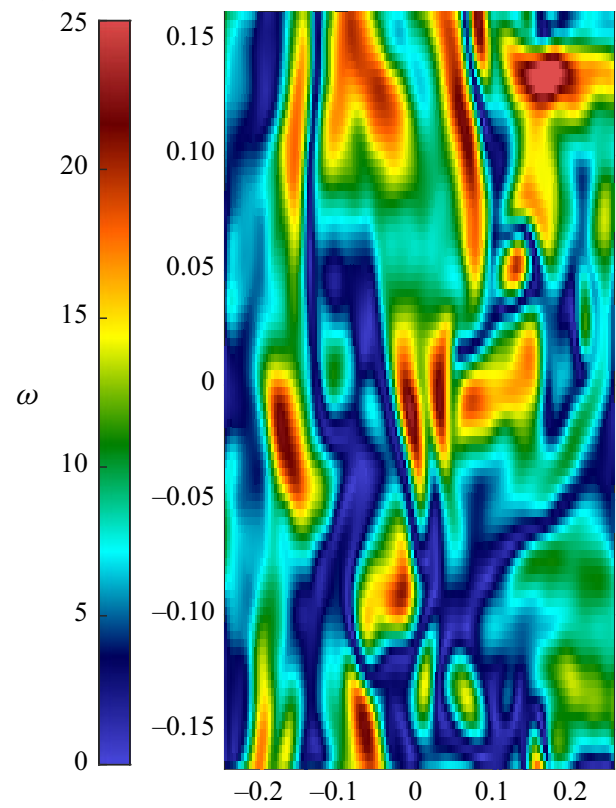

(b)

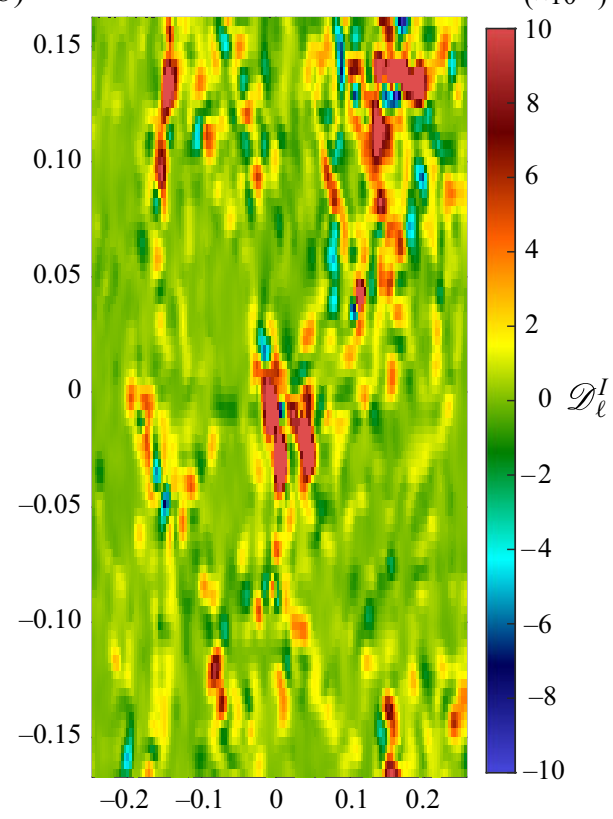

Figure 1. Visualization of the vorticity amplitude $\omega(a)$ and $\mathscr{D} I(b)$ for $\ell=3.2 \eta$ for the case T- 4 of table 1 in a plane containing the cylinder's axis.

(a)

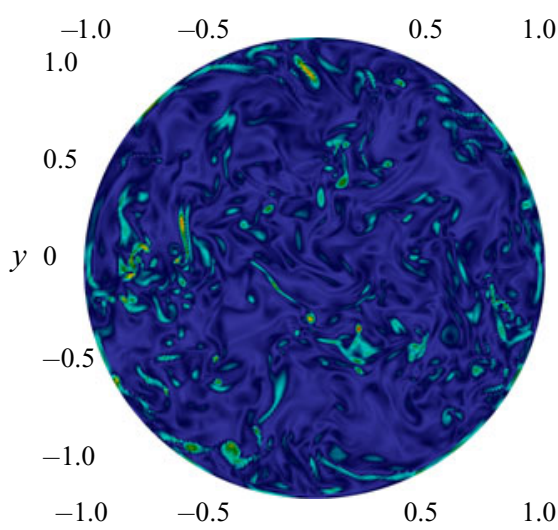

(b)

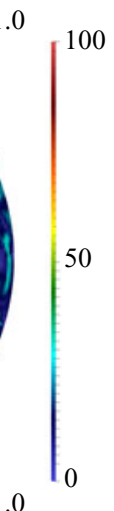

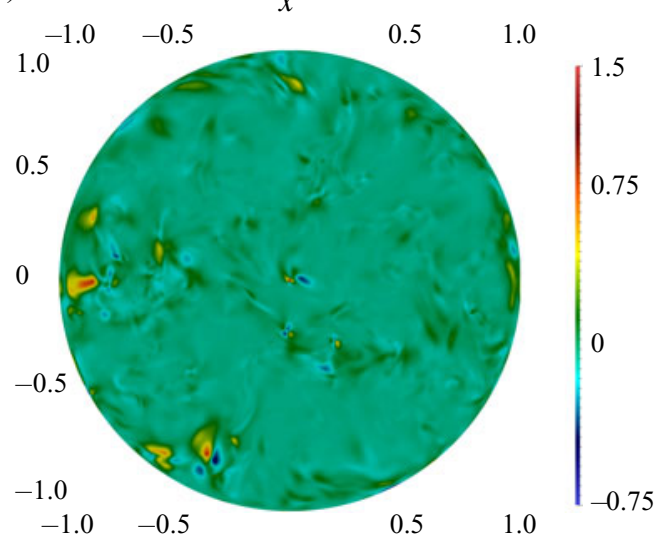

Figure 2. Visualization of the vorticity amplitude of $\omega(a)$ and $\mathscr{D}_{\ell}^{I}(b)$ for $\ell=8 \eta$ on a plane containing the centre perpendicular to the cylinder's axis from the DNS of table 1 .

measurement at scale $\ell=3.2 \eta$ in the case T-4, while figure 2 comes from DNS data. As table 1 shows, these two cases share the same Reynolds number and resolution.

Despite a higher level of noise in the local energy transfer fields, one observes a clear but not exact spatial correlation between location of high vorticity amplitude and high values of $\mathscr{D}_{\ell}^{I}$, as already noted in Saw et al. (2016). In figure 2, we show an equivalent comparison between an instantaneous vorticity field and the local energy transfer, in a mid-height plane perpendicular to the rotation axis. As in the experimental case, we observe a spatial correlation between local maxima of vorticity and local energy transfer, but the correlation 


\section{On the nature of intermittency in a von Kármán flow}

is not exact. To quantify further such correlation, we perform in the next section a detailed statistical analysis.

\subsection{Joint statistics}

Figure 3 (respectively figure 4) shows the joint probability distribution function (PDF) between the amplitude of the vorticity $\omega$ and the local energy transfer $\mathscr{D}_{\ell}^{I}$ at different scales, in the experiment T-4 (respectively in the DNS). Each PDF is computed over the available dataset which corresponds to the experimental cuboid for the experiment T-4, and to the entire tank, excluding the impellers for the DNS. Except for very large values of $\omega /\langle\omega\rangle$ and $\mathscr{D}_{\ell}^{I} / \epsilon$, all cases display a pyramidal correlation, similar to what was observed in Debue (2019) for the joint PDF between the local dissipation and the local energy transfer $\mathscr{D}_{\ell}^{I}$. This is not surprising, since dissipation and enstrophy are known to be correlated. The joint PDF of $\left(\mathscr{D}_{\ell}^{I} / \epsilon, \omega /\langle\omega\rangle\right)$ shows that, for every scale, strong events of inter-scale transfer are associated with strong vorticity. In the inertial range (see figure $3 b$, respectively figure $4 b), \ell \gg \eta$, the PDF is tilted towards positive energy transfers, indicating that the energy is going down the scales. In the viscous range (see figure $3 a$, respectively figure $4 a$ ), the PDF seems to be symmetric with respect to the line $\mathscr{D}_{\ell}^{I}=0$. A marked discrepancy appears between the numerical results and the experiments when looking at high values of $\omega /\langle\omega\rangle$, since the joint PDF computed from the DNS exhibits a well-defined elongated feature, tracing a high degree of correlation between $\omega /\langle\omega\rangle$ and $\mathscr{D}_{\ell}^{I} / \epsilon$. We have investigated the spatial distribution of the events corresponding to this tail in an instantaneous snapshot. It is displayed in figure 5. We see that these events are organized into coherent structures, with three favoured locations: (i) at the blade forefront, in the impeller region, where each blade strongly pushes the flow; (ii) at the outer edge of the disk supporting the impeller; (iii) in two blobs that are lying near the cylinder edge, and just above or just below the middle shear layer. While the first two types of event can be associated with local gradient sources due to the impellers that are difficult to measure experimentally, the last category corresponds to the location of the strong vortices of the shear layer. The latter are known to be present in the von Kármán geometry (Ravelet, Chiffaudel \& Daviaud 2008) and they are the locus of strong energy transfers (Marie \& Daviaud 2004; Kuzzay, Faranda \& Dubrulle 2015). This explains why such events have both strong vorticity, and strong local energy transfer. The absence of strong correlated events in the middle of the tank may explain why the joint $\operatorname{PDF} \mathbb{P}\left(\mathscr{D}_{\ell}^{I} / \epsilon, \omega /\langle\omega\rangle\right)$ based on experiment T-4 does not exhibit the tail, since the corresponding data were only taken in a small cube at the centre of the tank.

Using the joint PDF, we have computed the conditional average $\mathbb{E}\left(\mathscr{D}_{\ell}^{I} / \epsilon \mid \omega /\langle\omega\rangle\right)$ as the average of $\mathscr{D}_{\ell}^{I} / \epsilon$ over the points where $\omega /\langle\omega\rangle$ is fixed. It is plotted on figure 6 as a function of $(\omega /\langle\omega\rangle)^{2}$ both in the numerical and experimental cases. For $\ell \sim \eta$, the conditional average $\mathbb{E}\left(\mathscr{D}_{\ell}^{I} / \epsilon \mid \omega /\langle\omega\rangle\right)$ increases almost linearly with $(\omega /\langle\omega\rangle)^{2}$. For large $\ell \gg \eta$ the conditional averages seem to reach a plateau where the local energy transfers do not depend anymore on the vorticity, meaning that there is no correlation at large scales. This feature is more pronounced in the DNS than in the experiment. This shows that local energy transfers and vorticity are only correlated at small scales.

In order to investigate the link between the energy transfers and velocity increments, the joint PDF of $\mathscr{D}_{\ell}^{I}$ with the full $\left(\delta W_{\ell}\right)$ and anti-symmetric $\left(\delta \Omega_{\ell}\right)$ components of the velocity increments are plotted in figures 7 and 8 . It appears that positive values of $\mathscr{D}_{\ell}^{I}$ are promoted for both quantities. In the inertial range, it seems that the large $\mathscr{D}_{\ell}^{I}$ are more 


\section{H. Faller and others}

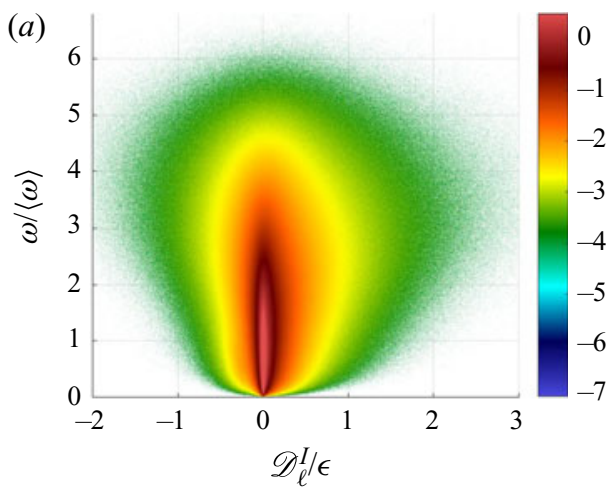

(b)

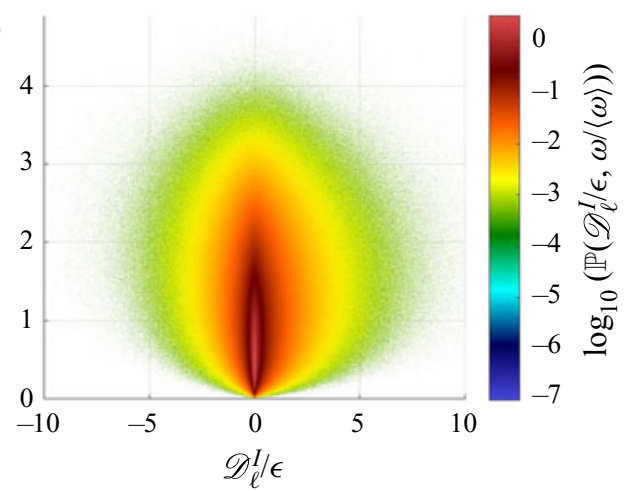

Figure 3. Joint PDF of $\mathbb{P}\left(\mathscr{D}_{\ell}^{I} / \epsilon, \omega /\langle\omega\rangle\right)$ for different scales from experimental measurements in table 1 computed over several uncorrelated snapshots. Here, $\omega$ refers to the norm of the vorticity, and $\mathscr{D}_{\ell}^{I}$ is the energy transfer. $(a) \mathrm{T}-4: \ell=3.2 \eta\left(3 \times 10^{4}\right.$ snapshots). $(b) \mathrm{T}-2: \ell=17.9 \eta\left(1.02 \times 10^{4}\right.$ snapshots $)$. White colour corresponds to a lack of events in the dataset.
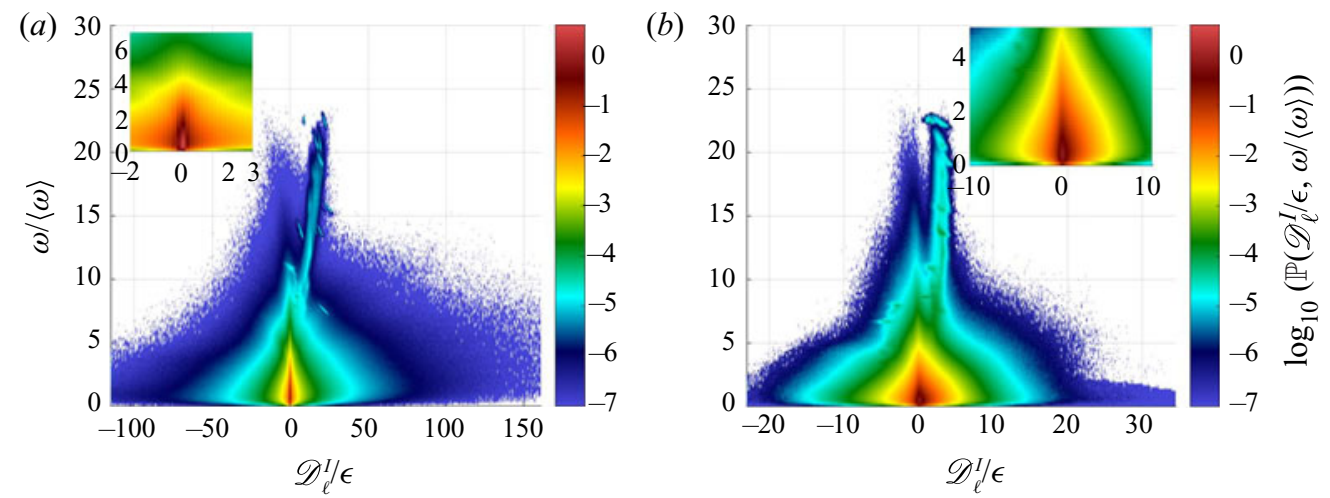

Figure 4. Joint PDF of $\mathbb{P}\left(\mathscr{D}_{\ell}^{I} / \epsilon, \omega /\langle\omega\rangle\right)$ for different scales from the DNS in table 1 computed over 21 uncorrelated snapshots. Here, $\omega$ refers to the norm of the vorticity, and $\mathscr{D}_{\ell}^{I}$ is the energy transfer. A zoom on the central region is presented in each panel to compare with figure 3. (a) $\ell=1.06 \eta ;(b) \ell=26.5 \eta$. White colour corresponds to lack of events in the dataset.

correlated with strong events of $\delta \Omega_{\ell}$ than with $\delta W_{\ell}$, since the PDF of $\left(\mathscr{D}_{\ell}^{I}, \delta \Omega_{\ell}\right)$ is more tilted to the right-hand side, and most of the positive energy transfers are associated with increments of the order of several times the mean value.

\subsection{Local energy transfers}

We now focus on the scale behaviour of the statistical average of the local energy transfer $\mathscr{D}_{\ell}^{I}$, and the local energy dissipation $\mathscr{D}_{\ell}^{v}$. The comparison between numerical and experimental data is performed in figure $9(a)$. For each experimental dataset, a filtering process is applied to the data giving a range of accessible $\ell$ scaling from $\Delta x$ to approximately $10 \Delta x$ depending on the size of the PIV grid. Combining all datasets with accessible $R_{\lambda}, \epsilon$ and $\eta$ allows us to cover a wide range of scales in terms of $\ell / \eta$. They all behave according to the Kolmogorov 1941 phenomenology (Dubrulle 2019): the local energy transfer is much lower than the local energy dissipation for $\ell / \eta<10$ and saturates beyond a constant value, while the local energy dissipation decreases 


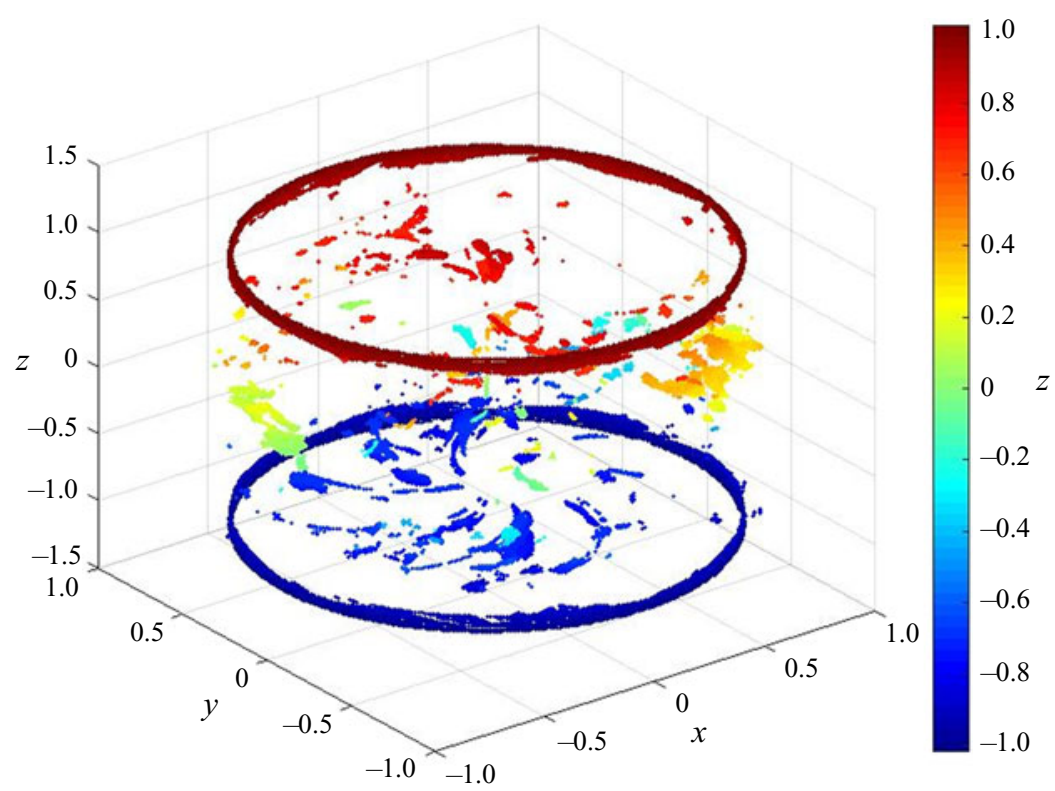

Figure 5. Positions of DNS points in the tail of figure $4(b)$ where $\mathscr{D}_{\ell}^{I}>\epsilon$ and $\omega>5\langle\omega\rangle$ for $\ell=26.5 \eta$. The colours correspond to the $z$ values of the points, indicating that most of them are close to the impellers $(0.7<$ $|z|<1.02$ ), or at the tank mid-height. This plot shows positions for one single snapshot.
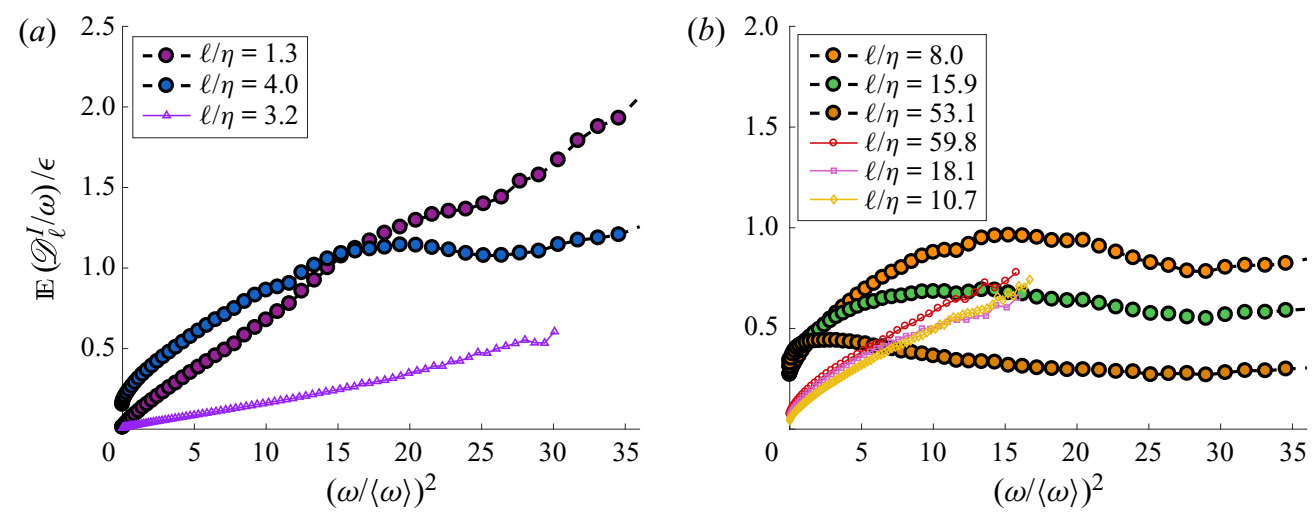

Figure 6. Conditional average $\mathbb{E}\left(\mathscr{D}_{\ell}^{I} \mid \omega\right) / \epsilon$ as a function of $(\omega /\langle\omega\rangle)^{2}$ for different scales $\ell$. They are computed from joint PDFs as in figures 3 and 4 from datasets of the DNS and cases T-1 to T-4 in table 1. Symbols are coded according to table 1 .

from the dissipative range to the large scales following a $\ell^{-4 / 3}$ law. The agreement between experimental data and numerical data is very good for $\mathscr{D}_{\ell}^{v}$, but poor for $\mathscr{D}_{\ell}^{I}$. We assign such mismatch to convergence effects: indeed, even if our statistical samples are quite large, the presence of very large events in the local transfer that can be either positive or negative renders the statistics very difficult to converge. Note that $\mathscr{D}_{\ell}^{v}$ is mainly positive, so that the plots in figure $9(a)$ of $\left\langle\mathscr{D}_{\ell}^{v}\right\rangle$ and figure $9(b)$ of $\left\langle\left|\mathscr{D}_{\ell}^{v}\right|\right\rangle$ are almost the same. These plots show that the agreement is excellent between experimental and DNS data. In contrast, $\mathscr{D}_{\ell}^{I}$ takes extreme positive and negative values. We suspect that this is the reason why the statistics of $\left\langle\mathscr{D}_{\ell}^{I}\right\rangle$ in figure $9(a)$ are less well 


\section{H. Faller and others}
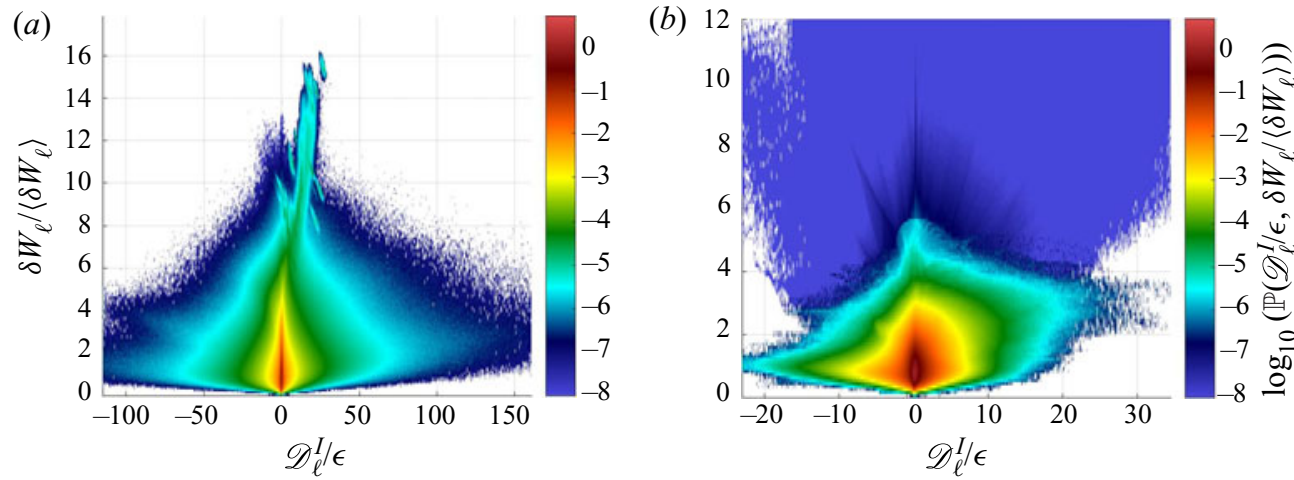

Figure 7. Joint PDF of $\mathbb{P}\left(\mathscr{D}_{\ell}^{I} / \epsilon, \delta W_{\ell} /\left\langle\delta W_{\ell}\right\rangle\right)$ for different scales from the DNS in table 1 computed over 21 uncorrelated snapshots. $(a) \ell=1.06 \eta ;(b) \ell=26.5 \eta$. White colour corresponds to lack of events in the dataset.
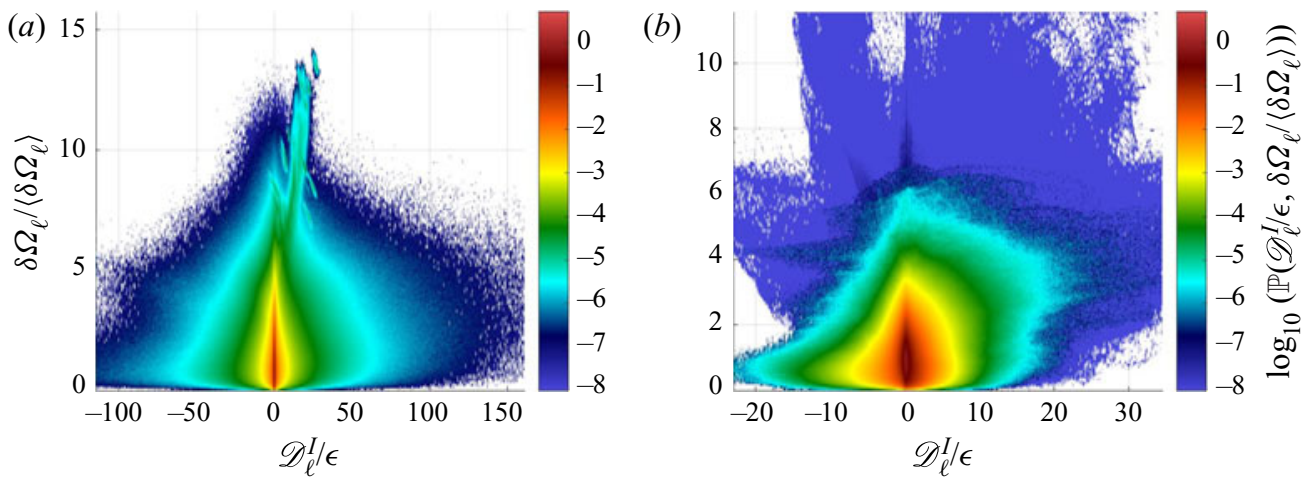

Figure 8. Joint PDF of $\mathbb{P}\left(\mathscr{D}_{\ell}^{I} / \epsilon, \delta \Omega \ell /\langle\delta \Omega \ell\rangle\right)$ for different scales from the DNS in table 1 computed over 21 uncorrelated snapshots. (a) $\ell=1.06 \eta ;(b) \ell=26.5 \eta$. White colour corresponds to lack of events in the dataset.

converged. To test this idea, we plot in figure $9(b)\left\langle\left|\mathscr{D}_{\ell}^{I}\right|\right\rangle$, which shows that statistics are indeed improved. This issue is linked to the presence of rare events of extreme values of $\mathscr{D}_{\ell}^{I}$, as discussed in the appendix of Debue et al. (2018) for experimental points. A convergence study of the DNS statistics is presented in the appendix A (figure 15). On the other hand, the local dissipation, that is always positive, does not suffer from such drawback. To check such a hypothesis, we plot in figure $9(b)$ the average of the absolute values of $\mathscr{D}_{\ell}^{I}$ and $\mathscr{D}_{\ell}^{v}$. Indeed, we see that the data are less scattered, and that data points corresponding to different experiments follow a clear trend. The numerical data are closer to the TPIV data than the SPIV data. This might be due to the fact that SPIV data are 2-D-3C, meaning that some components of $\mathscr{D}_{\ell}^{I}$ are missing. These plots show that the 'inertial range' (location where $\mathscr{D}_{\ell}^{I}$ is constant) extends from $\ell / \eta=20$ to $\ell / \eta=100$ for the numerical data. Such observation will be used to compute the scaling exponents in $\S 4.6$.

\subsection{Structure functions}

Longitudinal and wavelet velocity structure functions, as well as non-dimensional moments of $\mathscr{D}_{\ell}^{I}$, have been previously computed and discussed in Saw et al. (2018), 

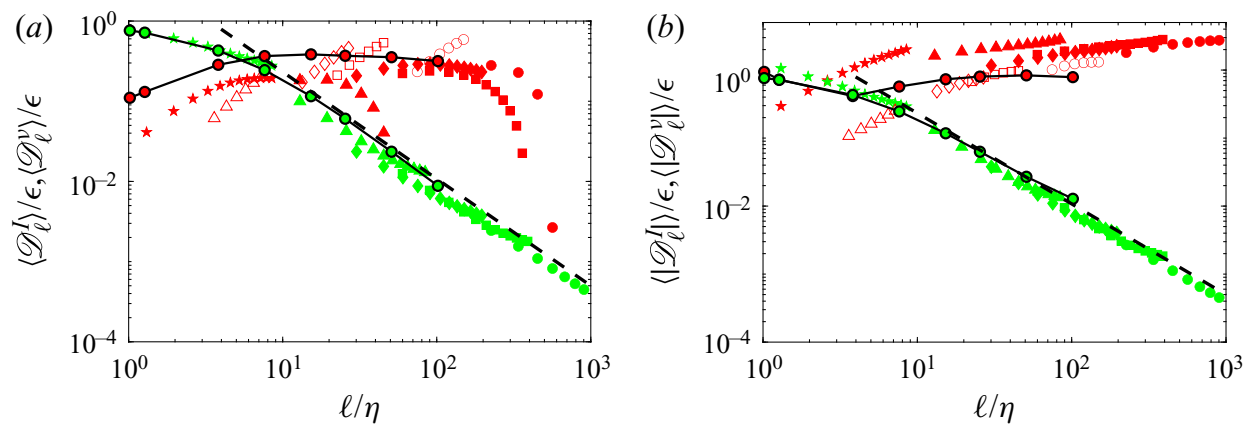

Figure 9. Scale variation of the non-dimensional local energy transfer (red) and dissipation (green) for SPIV experiments A to E (filled symbols), TPIV experiments (open symbols) and the DNS (filled symbols with black line). Dotted lines correspond to $\ell^{-4 / 3}$. Panel $(a)$ for $\left\langle\mathscr{D}_{\ell}^{I}\right\rangle$ and $\left\langle\mathscr{D}_{\ell}^{v}\right\rangle$ non-dimensionalized by the total energy dissipation in the observational box; $(b)$ same for absolute values. The symbols are coded according to table 1 .
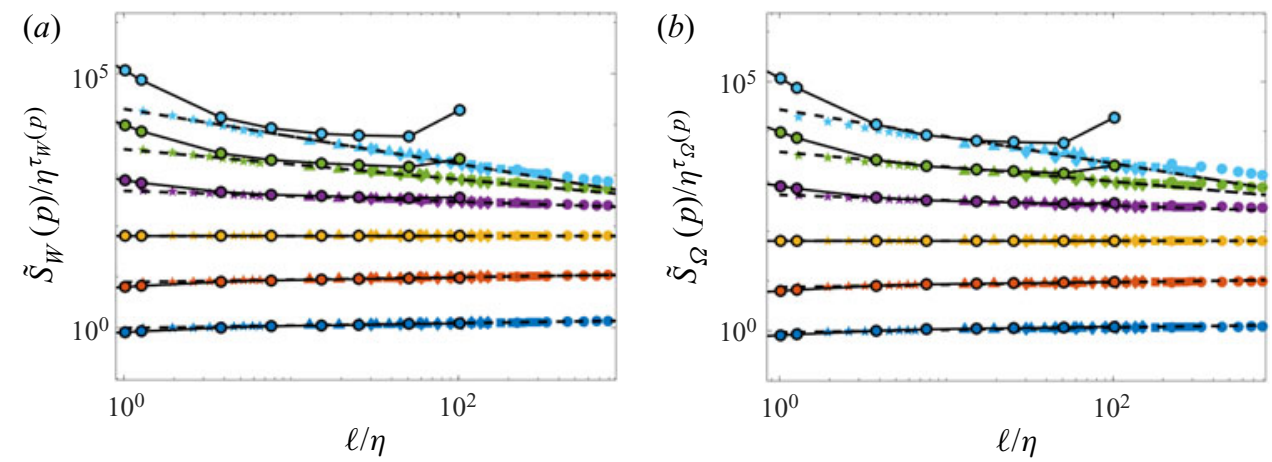

Figure 10. Scale variation of the normalized non-dimensional wavelet structure functions of order $p=1$ to $p=6$ for SPIV experiments A to E (filled symbols) and the DNS (filled symbols with black line) The structure functions have been shifted by arbitrary factors for clarity and are coded by colour: $p=1$, blue symbols; $p=2$, orange symbols; $p=3$, yellow symbols; $p=4$, magenta symbols; $p=5$, green symbols; $p=6$, light blue symbols. (a) Structure functions $\tilde{S}_{W}(p)$. (b) Structure functions for the anti-symmetric component $\tilde{S}_{\Omega}(p)$. The dashed lines are power laws with exponents $\tau_{W}$ and $\tau_{\Omega}$ shown in figure 13(a). The symbols are coded according to table 1 .

Debue et al. (2018) and Dubrulle (2019) from the experimental SPIV datasets A-E of table 1, with the drawback that the velocity field is only 2-D-3C. Here, we repeat their computation with experimental and numerical 3-D-3C data, with a focus on the wavelet velocity structure functions that are more appropriate for our purpose, see $\S 2.2$. Figure $10(a)$ shows the comparison of the compensated wavelet velocity structure functions $\tilde{S}_{W}(p)=\left\langle\delta W_{\ell}^{p}\right\rangle /\left\langle\delta W_{\ell}^{3}\right\rangle^{p / 3}$ of orders 1 to 6 for numerical and experimental SPIV data. One sees that they are in good agreement for $p=1$ to 4 , and that the agreement deteriorates at large scales for higher $p$. This is probably due to a lack of convergence for the numerical data because of limited sampling. The same comparison has been done for the anti-symmetric wavelet velocity structure functions in figure $10(b)$, leading to a similar conclusion.

Finally, we compare the compensated moments of the local energy transfer $\mathscr{D}_{\ell}^{I}$ for numerical and experimental data in figure 11(a). There is good agreement between 


\section{H. Faller and others}

(a)

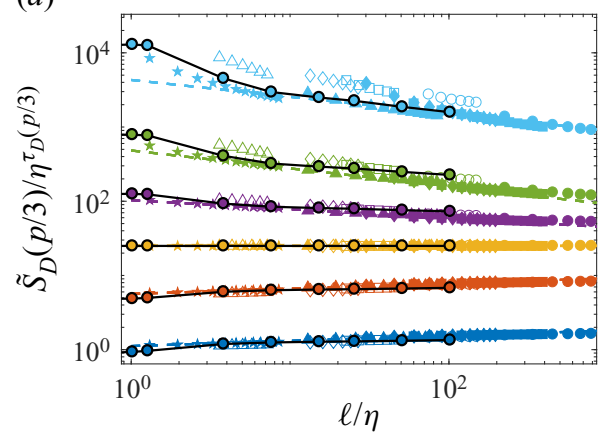

(b)

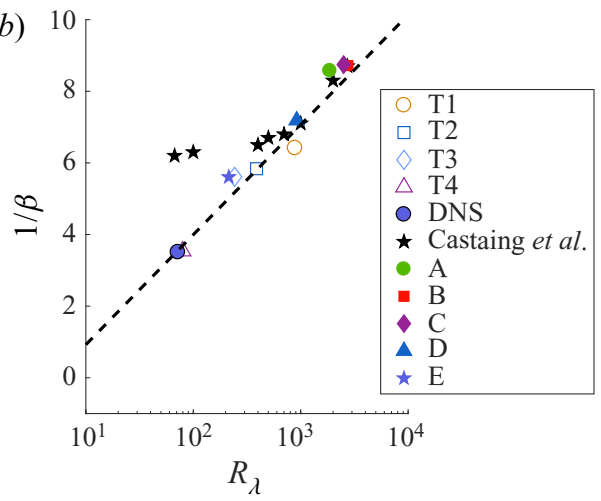

Figure 11. (a) Scale variation of the compensated structure functions of the local energy transfer $\tilde{S}_{D}(p / 3)$ of orders $p=1$ to 6 for SPIV experiments A to E (filled symbols), TPIV experiments (open symbols) and the DNS (filled symbols with black line). The structure functions have been shifted by arbitrary factors for clarity and are coded by colour: $p=1$, blue symbols; $p=2$, orange symbols; $p=3$, yellow symbols; $p=4$, magenta symbols; $p=5$, green symbols; $p=6$, light blue symbols. The dashed lines are power laws with exponents $\tau_{D}$ shown in figure 13(a). The symbols are coded according to table 1. (b) Parameter $\beta$ given in (4.1). Labels are taken according to table 1 . The dotted line follows the equation $1 / \beta=(4 / 3) \log \left(R_{\lambda}\right)$.

numerical data and experiments in the inertial range, but not in the dissipative range or at large scales.

\subsection{Test of log universality}

In figure 11(a), we compare the structure functions of the local energy transfer using data at different Reynolds numbers (see table 1). In agreement with the Kolmogorov self-similarity hypothesis, we can expect, and we indeed observe, that they have a universal behaviour in the inertial range, but not outside. As first discussed by Frisch \& Vergassola (1991) and Castaing, Gagne \& Hopfinger (1990), a more general log-universality property can be expected using the multi-fractal hypothesis, if one works with variables that are rescaled by a factor proportional to $\log \left(R_{\lambda}\right)$, where $R_{\lambda}$ is the Taylor Reynolds number. In Geneste et al. (2019), we have indeed shown that such a rescaling enables a better collapse of the velocity structure functions $S_{W}$, and linked such log universality with the extensivity of the large deviation function of the multi-fractal measure $\delta W_{\ell}^{3} /\left\langle\delta W_{\ell}^{3}\right\rangle$. If the local refined similarity hypothesis (2.9) holds true, one can expect that the log universality also applies to the measure $\left|\mathscr{D}_{\ell}^{I}\right| /\left\langle\left|\mathscr{D}_{\ell}^{I}\right|\right\rangle$, so that

$$
\beta(\operatorname{Re})\left(\frac{\ln \left(\tilde{S}_{D}(p) / S_{0 p}\right)}{\ln \left(L_{0} / \eta\right)}\right)=F\left(p, \beta(\operatorname{Re}) \frac{\ln (\ell / \eta)}{\ln \left(L_{0} / \eta\right)}\right) .
$$

We take the DNS at $R_{\lambda}=72$ as the reference case, and find, for both DNS and experiments, values of $\beta(R e)$ and $S_{0 p}$ that best collapse the curves. The corresponding collapses are provided in figure 12. The collapse is good for any value, except for the TPIV at the lowest Reynolds number, which does not collapse in the dissipative range. The collapsing curves display three different scaling regimes. At low $\ell / \eta$, a saturation can only be seen in the DNS, which corresponds to the far viscous range, where the velocity field becomes regular, so that $\left\langle\left|\mathscr{D}_{\ell}^{I}\right|^{p}\right\rangle \sim \ell^{2 p}$ (Dubrulle 2019), and $S_{D}(p) \sim$ cte. In the inertial range, we observe a scaling $S_{D}(p) \sim \ell^{\tau_{D}}(p)$ and in between, an intermediate range due to 
On the nature of intermittency in a von Kármán flow

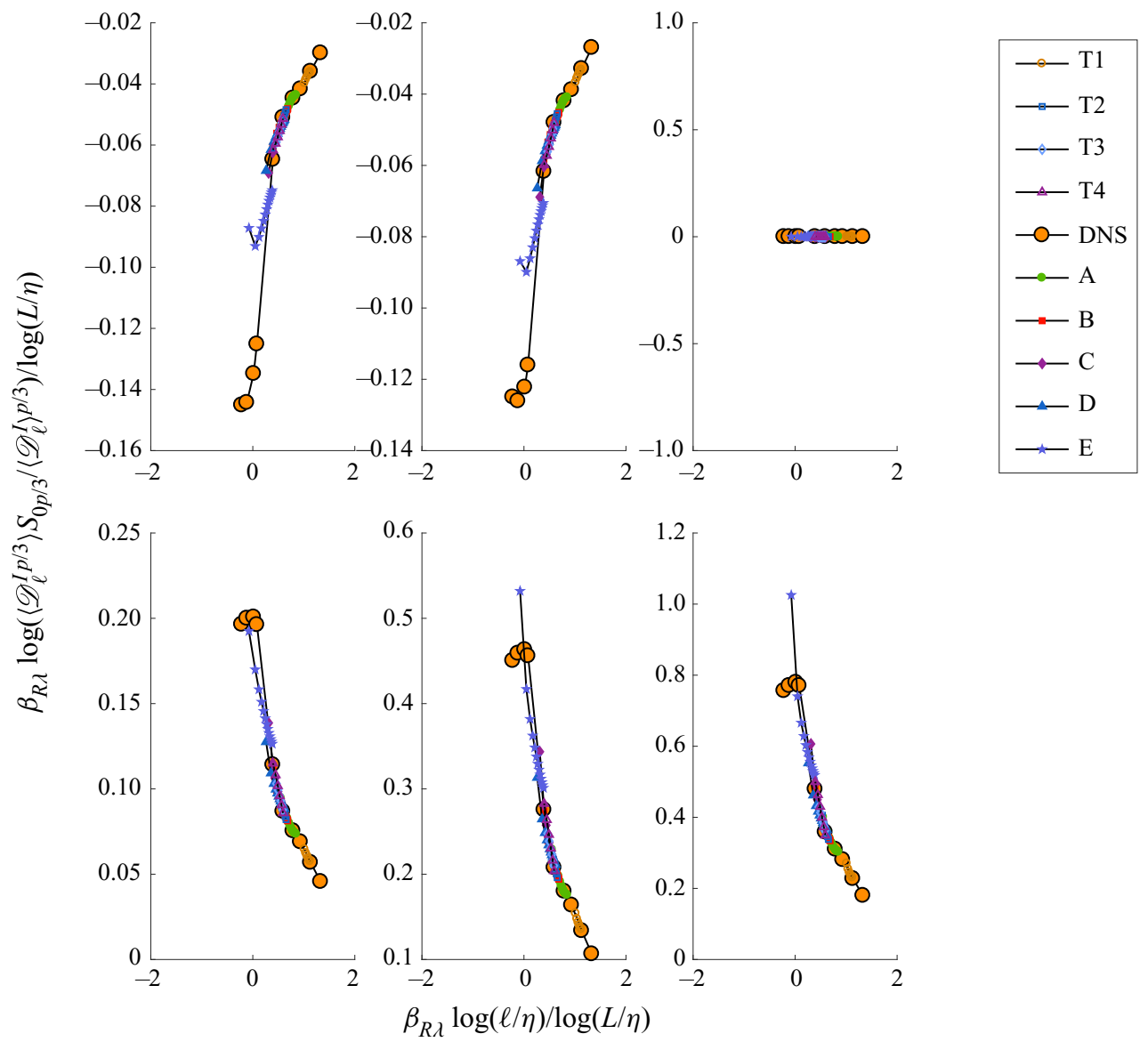

Figure 12. Computation of (4.1) from order $p=1$ to order $p=6$. Collapse is observed for all experiments presented in table 1, using DNS (orange circles) as the reference and the $\beta$ parameter taken from figure 11(b).

the random character of the dissipative scale, corresponding to the coexistence of regions of flow with different Hölder exponents, with areas where the flow has been relaminarized due to the action of viscosity (Geneste et al. 2019).

The values of $\beta(R e)$ are shown in figure $11(b)$. In agreement with previous results (Geneste et al. 2019), they collapse on a curve $1 / \beta \sim\left(1 / \beta_{0}\right) \log \left(R_{\lambda}\right)$, with $\beta_{0} \sim 3 / 4$ over the whole range of Reynolds numbers, and we do not observe the saturation of $1 / \beta$ at low Reynolds numbers that is observed in the jet experiment of Castaing, Gagne \& Marchand (1993).

\subsection{Scaling exponents and multi-fractal spectrum}

Using the identification of the inertial range based on the local energy transfer (see $\S 4.3)$, we compute from figures 10 and 11 the scaling exponents $\tau_{W}(p), \tau_{\Omega}(p)$ and $\tau_{D}(p / 3)$ for the numerical data. They are shown in figure 13(a). We see that $\tau_{W}(p)$ and $\tau_{\Omega}(p)$ overlap in the range $p \in[-5,5]$, and both overlap with $\tau_{D}(p / 3)$ in the range $p \in[0,5]$, thereby validating the refined similarity hypothesis expressed by (2.9) (Dubrulle 2019). These overlapping values computed from numerical data compare very well with the value $\tau_{W}(p)$ computed on the SPIV data by Debue et al. (2018) and Dubrulle (2019). 


\section{H. Faller and others}
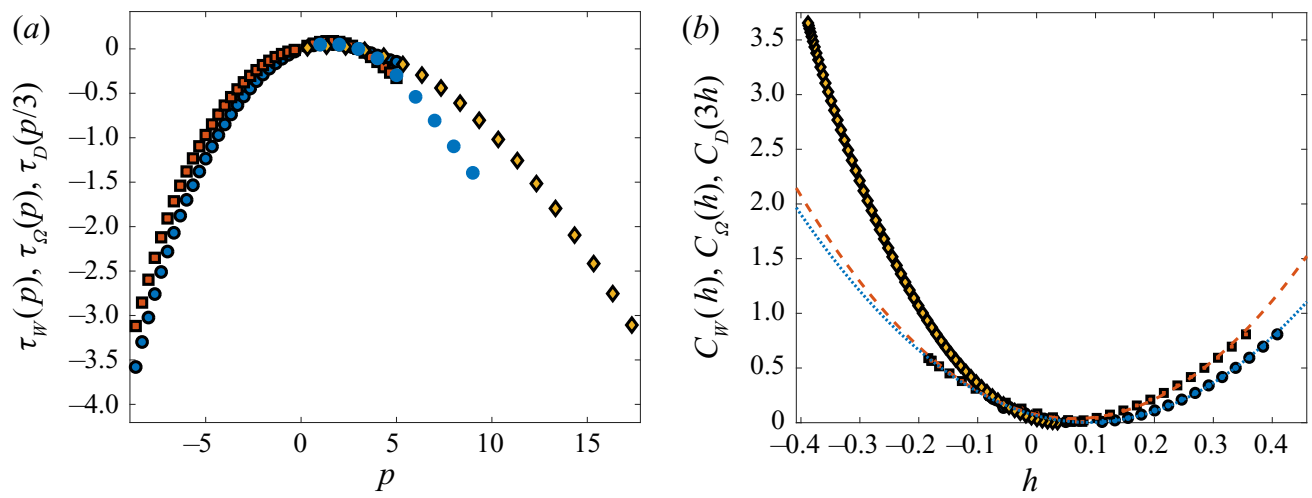

Figure 13. (a) Scaling exponents as a function of order for DNS (filled symbols with black outside) and SPIV (filled symbols): $\tau_{W}$, blue circles; $\tau_{\Omega}$, red squares; $\tau_{D}$, yellow diamonds. (b) Corresponding multi-fractal spectrum $C(h)$ for the DNS scaling exponents. The spectrum has been obtained by taking the inverse Legendre transform of the scaling exponents $\tau(p)$ shown in figure 13(a). The dotted line curves are parabolic fit $C(h)=$ $a h^{2}+b h+c$ with $a=8$ (respectively 9.5 ), $b=-1.4$ (respectively -1.2 ) and $c=0.064$ (respectively 0.075 ) for $C_{W}$ (respectively $C_{\Omega}$ ).

We checked that the values of $\tau_{D}(p / 3)$ coincide with the values obtained using figure 12 . We also completed our measurements by computing more positive and negative moments, so as to be able to better estimate the multi-fractal spectra $C_{W}(h), C_{\Omega}(h)$ and $C_{D}(h)$ by Legendre transforms (see (2.13)). The resulting spectra are shown in figure 13(b). The two spectra $C_{W}(h)$ and $C_{\Omega}(h)$ are parabolic, with a minimum shifted from 0 by approximately $\delta h=0.08$. They are very close to each other for $h<0$ but differ more markedly for $h>0$, implying a different topology of regular regions. The value $C_{W}(h)=3$ or $C_{\Omega}(h)=3$ extrapolated from the parabolic fit results in $h_{\min } \approx-0.53$. Using the shift property (2.14) and the measurement of $\zeta_{W}(3)=0.8$ (Dubrulle 2019), we can then estimate the most probable exponent for $\zeta_{W}$ and $\zeta_{\Omega}$ as 0.35 , very close to the Kolmogorov value 0.33 . The minimum exponent for $C(h)$ is then $h_{\min }=-0.26$. Note that the multi-fractal spectrum of the local energy transfer $C_{D}(3 h)$ deviates strongly from the parabolic fit of $C_{W}(h)$, and displays a milder intermittency, as it is more peaked around the most probable value. This is not caused by insufficient statistics as the values of $\tau_{D}(p / 3)$ are converged (see appendix A). It could be caused either by finite resolution (the finer scale is not small enough), meaning that we missed some very large energy transfers (Dubrulle 2019), or by a genuine difference between intermittency at very small scales caused by vorticity and the local energy transfer. To try to understand further the origin of intermittency, we then attempt another approach, based on conditional statistics.

\subsection{Conditioned scaling exponents}

To understand further the origin of intermittency, it is natural to condition the structure functions to areas of different values of the local energy transfer. An earlier attempt in that direction has been done in Debue et al. (2018), for the SPIV data, with the limitation that the conditioning could only be done on the local energy transfer at the smallest available scale of the dataset. Therefore, the conditioning was performed at the Kolmogorov scale only for the dataset $\mathrm{E}$. The conclusion of such conditioning was that the intermittency was higher for areas corresponding to a larger (in absolute value) local energy transfer.

Using the data from the DNS, we can improve the procedure, by conditioning the structure functions at all scales by the local energy transfer at the Kolmogorov scale 
(a)

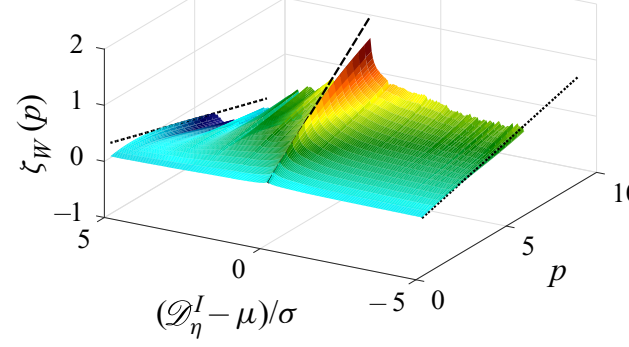

(b)

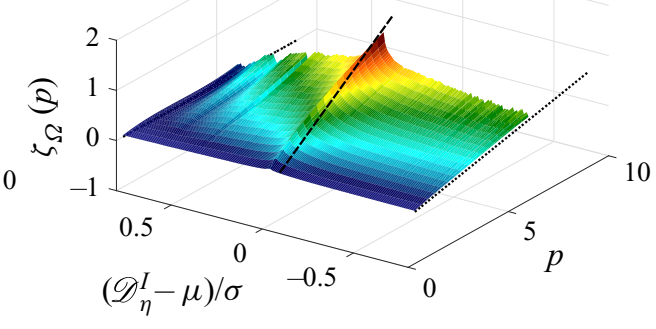

Figure 14. (a) Scaling exponent of the non-compensated wavelet structure functions $S_{W}$ computed using the joint PDF of the centred and reduced value of $\mathscr{D} I I$ and $\delta W_{\ell}$ at various scales $\ell$, and then a fit in the inertial range. The dotted lines have a slope of -0.1 for large positive values of centred and reduced value of $\mathscr{D}_{\eta}^{I}, 1 / 3$ for small values of centred and reduced value of $\mathscr{D}_{\eta}^{I}$ and 0.1 for large negative values of centred and reduced value of $\mathscr{D}_{\eta}^{I}$. (b) Same as (a), using the joint PDF of the centred and reduced value of $\mathscr{D}_{\eta}^{I}$ and $\delta \Omega_{\ell}$. The dotted lines have a slope of 0 for large positive values of centred and reduced value of $\mathscr{D}_{\eta}^{I}, 1 / 3$ for small values of centred and reduced value of $\mathscr{D}_{\eta}^{I}$ and 0.1 for large negative values of centred and reduced value of $\mathscr{D}_{\eta}^{I}$.

$\mathscr{D}_{\eta}^{I}$. Here, we enlarge the discussion by considering positive and negative values of the local energy transfer, as they may correspond to a different dynamics. Because of the conditioning, we have less statistics so we have to restrict ourselves to smaller values of $p$ than for unconditioned values. Once the structure functions have been conditioned, we can compute the corresponding conditioned scaling exponent by fitting power laws in the inertial range. The results for $S_{W}$ are shown in figure 14(a), for $p$ ranging from 0 to 6 and for centred reduced values of $\mathscr{D}_{\eta}^{I}$ ranging from -5 to 5 . One sees that for values $\mathscr{D}_{\eta}^{I}$ close to the mean, the scaling exponents are positive, and close to the Kolmogorov value $p / 3$. For large negative $\mathscr{D}_{\eta}^{I}$, the scaling exponents are positive, but increase linearly with a small exponent $h_{-} \sim 0.1$. For large positive $\mathscr{D}_{\eta}^{I}$, the scaling exponents decrease quasi-linearly with a small exponent $h_{+} \sim-0.1$. This shows that negative local energy transfers are less intermittent than positive local energy transfers. The corresponding values of $h$ are close to 0 , reminiscent of shock-like structures. Exploring more singular structures closer to $h=-0.26$ (the minimum exponent of $C(h)$ ) would require a much larger number of statistics. We have repeated the analysis for the structure functions of the vortical part $S_{\Omega}$ in figure $14(b)$. We observe a similar trend for large negative and positive values of $\mathscr{D}_{\eta}^{I}$, where we see that the scaling exponents are much more intermittent than for $\mathscr{D}_{\eta}^{I}$ around its mean value. In both cases, the conditioned exponent displays linear trends with $h_{-} \sim 0.1$ and $h_{+} \sim 0$, exponents that are close to their counterpart using $S_{W}$. Such a linear trend might be an indication that only one type of very singular structure is responsible for the scaling behaviour of $S_{W}$ or $S_{\Omega}$ for large positive or negative local energy transfers.

\section{Discussion}

We have conducted an extensive study of the scaling properties of small scale turbulence using both numerical and experimental data of a flow in the von Kármán geometry. Our investigations validate both Kraichnan intuition, and Batchelor early observation: areas of strong vorticity are correlated to areas of strong local energy transfer; both are highly intermittent, and responsible for deviations of the structure functions from the Kolmogorov 1941 simple mono-fractal picture. Such findings are not surprising given the already 


\section{H. Faller and others}

known link between intermittency and dissipation (Kolmogorov 1962), on the one hand, and dissipation and enstrophy and dissipation and local energy transfer (Dubrulle 2019) on the other hand. These results show once more the necessity of dealing with the very small scales of turbulence when it comes to the modelling of realistic velocity fluctuations, a problematic issue when considering very high Reynolds number flows, in which the ratio of the integral to the dissipative scale goes like $\operatorname{Re}^{3 / 4}$.

On the other hand, the correlation we find between vorticity and local energy transfer is not perfect: in the joint PDF, we observe in figure 4 a stretched diamond shape for values of $\omega$ that are not too high, demonstrating that large values of local energy transfers are correlated with medium values of $\omega$, while areas of large $\omega$ are independent of local energy transfer. This is an indication that the locations of the local maxima of energy transfer are shifted with respect to the locations of the local maxima of vorticity, a fact already observed in our experiment (Debue 2019; Debue et al. 2020) and in numerical simulations (Nguyen et al. 2019). This points out the complex dynamics of the small scale structures of turbulence, and calls for further investigation, to determine the role of local energy transfer in the formation of high vorticity zones or the reverse.

Quite interestingly, we have nevertheless gathered several indications that, in the von Kármán flow, the most intermittent structures might have an interesting simple structure: first, the linear trend between the conditional average of $\left|\mathscr{D}_{\ell}^{I}\right|$ versus $\omega^{2}$ for scales small enough; second, the linear trend of the conditional scaling exponent of $S_{W}$ or $S_{\Omega}$, observed for high negative or positive values of the local energy transfer in our numerical data in figure 14; finally, the linear tails observed in the joint PDF between the vorticity and the local energy transfer in our numerical data (figure 4), that correspond partly to strong vortices of the shear layer. This location corresponds obviously to areas where the shear, hence the dissipation and the fluctuations, are maximum. In Marie \& Daviaud (2004), it was indeed found that most of the angular momentum transfer occurs in such zone via correlated azimuthal and vertical velocity fluctuations. Such a location (near the cylinder boundary, at the mid-height) is also the location where Luo \& Hou (2014) detected a potential singularity in an Euler axisymmetric flow with a geometry similar to the shear layer geometry. The blowing-up solution was furthermore shown to develop a self-similar structure near the point of the singularity, as the singularity time is approached.

Whether the vortices of the von Kármán shear layer we evidenced in figure 5 correspond to self-similar quasi-singularities of the Euler equations, that could dominate the scaling properties at large orders, is an interesting issue that is worth investigating further with time correlated snapshots. Indeed, they could act as the counterpart of the self-similar blowing solution of the inviscid Burgers equation or shell models that have been found recently to control the intermittency in such systems (Mailybaev 2015). To explore such an interesting connection, we need to follow in space and time the dynamics of such structures, which is both an experimental and numerical challenge, for different reasons (a noise issue in the first case, numerical burden in the second). Such studies are nevertheless underway.

Finally, it would also be interesting to perform a similar analysis of the link between areas of high energy transfers and large vorticities in other geometries, so as to explore whether our findings are due to the peculiar geometry of the von Kármán flow (with a shear layer and a stagnation point in the middle) or are linked to universal features of turbulent flows.

Acknowledgements. This work has been funded by the ANR, project EXPLOIT, grant agreement no. ANR-16-CE06-0006-01, and project ECOUTURB grant agreement no. ANR-16-CE30-0016. This work has also been founded by the Funding agency LabEx PALM Investissement d'Avenir under the grant number 


\section{On the nature of intermittency in a von Kármán flow}

ANR-10-LABX-0039-PALM. Access to the HPC resources were provided by IDRIS (institut du développement et des ressources en informatique scientifique) under the allocation 2019-0254 made by GENCI (Grand Equipement National de Calcul Intensif).

Declaration of interests. The authors report no conflict of interest.

\section{Author ORCIDs.}

(D) H. Faller http://orcid.org/0000-0002-2386-1028;

D V. Valori http://orcid.org/0000-0002-8010-295X;

(D) C. Nore http://orcid.org/0000-0001-9118-5247;

(D) B. Dubrulle http://orcid.org/0000-0002-3644-723X.

\section{Appendix A}

\section{A.1. DNS statistical convergence study}

The DNS data cover 21 uncorrelated snapshots of $3 \times 10^{8}$ grid points. To evaluate the convergence of the DNS data, we present here the empirical moments and mean values computed for an increasing number of snapshots. First of all, the averages $\left\langle\mathscr{D}_{\ell}^{I}\right\rangle$ and $\left\langle\mathscr{D}_{\ell}^{v}\right\rangle$ used in figure $9(a)$ are converged, as figure 15 shows. We can then focus on the moments of the statistical quantities. In the viscous range, for $\ell=1.06 \eta$ (figures 16a, 17a and 18a), the convergence is good for all $p$ since the statistics on 16 or 21 snapshots agree, and the 21 snapshot results lie between the 11 snapshot and 16 snapshot ones. In the inertial range, for
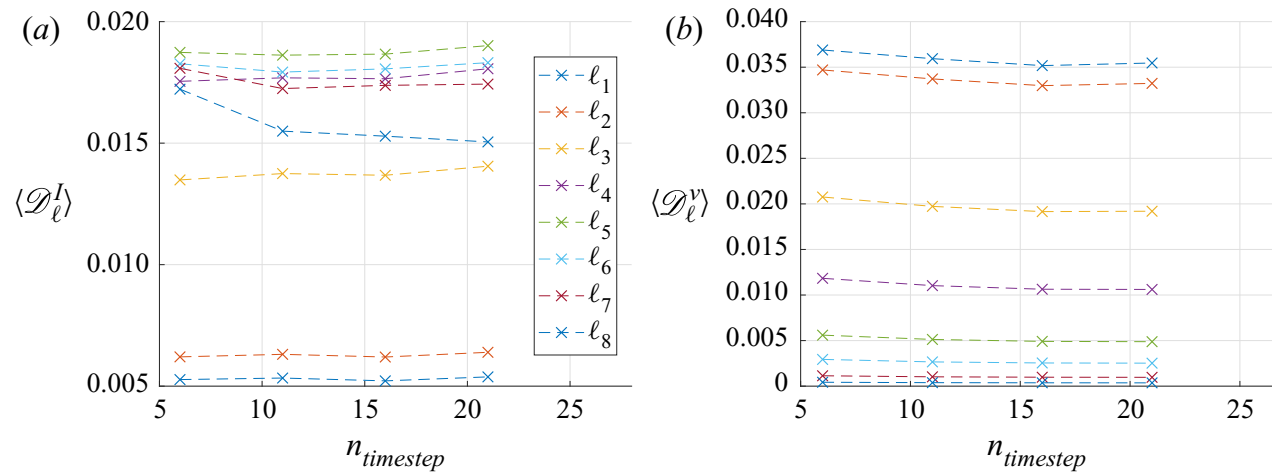

Figure 15. Convergence of mean DNS quantities for the 8 scales $\left(\ell_{1}<\ell_{2}<\cdots<\ell_{8}\right)$ presented in figure $9(a)$ computed on $6,11,16$ or 21 snapshots. $(a)\left\langle\mathscr{D}_{\ell}^{I}\right\rangle .(b)\left\langle\mathscr{D}_{\ell}^{v}\right\rangle$.

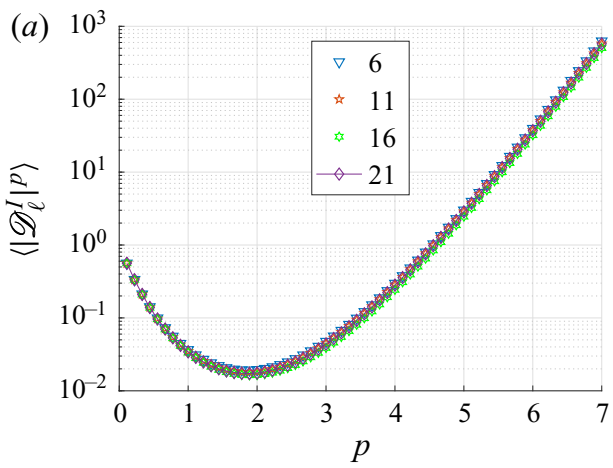

(b)

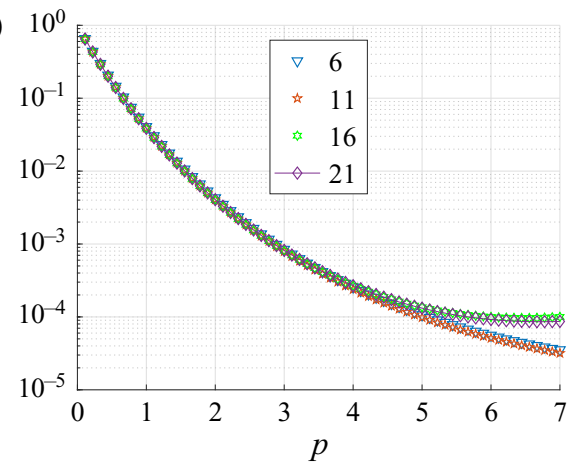

Figure 16. Moments of $\left|\mathscr{D}_{\ell}^{I}\right|$ computed on $6,11,16$ or 21 snapshots. $(a) \ell=1.06 \eta$. (b) $\ell=26.5 \eta$. 


\section{H. Faller and others}
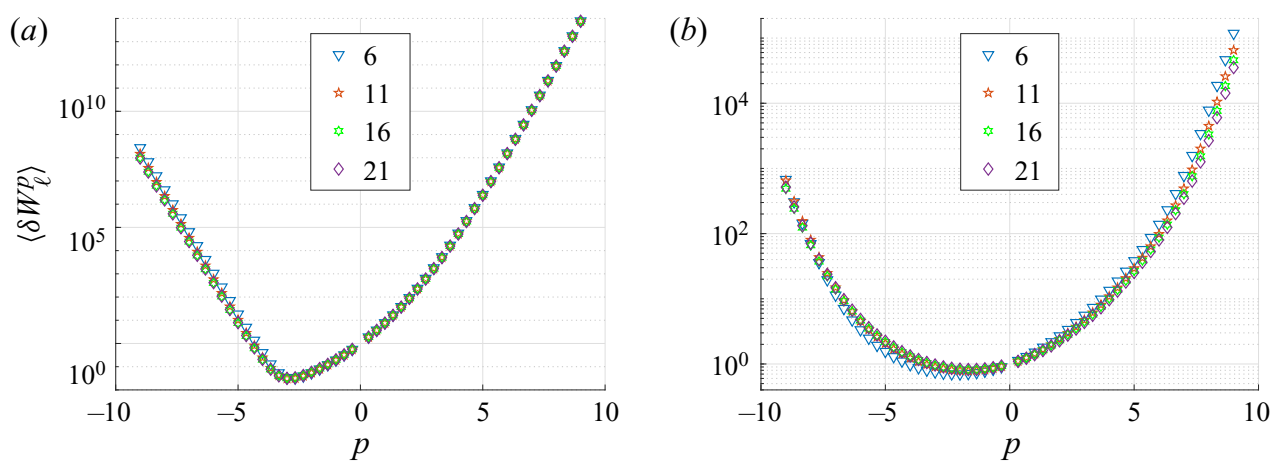

Figure 17. Moments of $\delta W_{\ell}$ computed on $6,11,16$ or 21 snapshots. (a) $\ell=1.06 \eta$. (b) $\ell=26.5 \eta$.
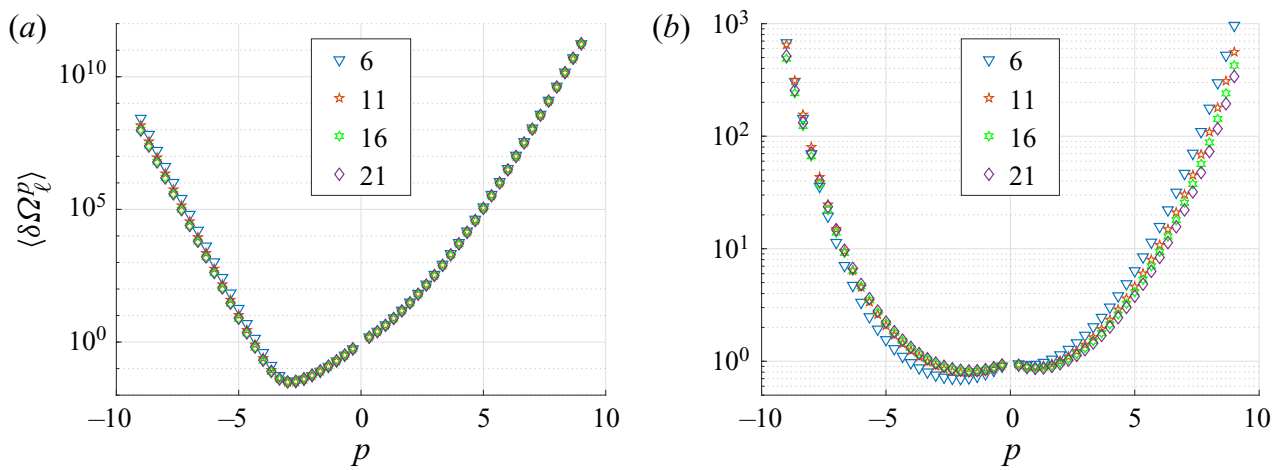

Figure 18. Moments of $\delta \Omega_{\ell}$ computed on $6,11,16$ or 21 snapshots. (a) $\ell=1.06 \eta$. (b) $\ell=26.5 \eta$.

$\ell=26.5 \eta$ (figures $16 b, 17 b$ and $18 b$ ), the moments are converged for the same reasons up to $p=5$, but not totally converged for $p \geq 5$ since the results do not coincide and decrease monotonically for velocity increments $\delta W_{\ell}$ and $\delta \Omega_{\ell}$ as the dataset size increases. Since the moments are used to compute the exponents $\tau(p)$, figure 13 only shows velocity and energy transfer scaling exponents up to $p=5$ for the DNS.

\section{REFERENCES}

Batchelor, G.K. \& Townsend, A.A. 1949 The nature of turbulent motion at large wave-numbers. Proc. R. Soc. Lond. A 199 (1057), 238-255.

Cappanera, L., Debue, P., Faller, H., Kuzzay, D., Saw, E.-W., Nore, C., Guermond, J.-L., Daviaud, F., Wiertel-Gasquet, C. \& Dubrulle, B. 2021 Turbulence in realistic geometries with moving boundaries: when simulations meet experiments. Comput. Fluids 214, 104750.

CAstaing, B., Gagne, Y. \& Hopfinger, E.J. 1990 Velocity probability density functions of high Reynolds number turbulence. Physica D 46 (2), 177-200.

Castaing, B., Gagne, Y. \& Marchand, M. 1993 Log-similarity for turbulent flows? Physica D 68 (3), 387-400.

Chainais, P., Abry, P. \& Pinton, J.-F. 1999 Intermittency and coherent structures in a swirling flow: a wavelet analysis of joint pressure and velocity measurements. Phys. Fluids 11 (11), 3524-3539.

Debue, P. 2019 Experimental approach to the problem of the Navier-Stokes singularities. PhD thesis, Université Paris-Saclay.

Debue, P., Shukla, V., Kuzzay, D., Faranda, D., Saw, E.-W., Daviaud, F. \& Dubrulle, B. 2018 Dissipation, intermittency, and singularities in incompressible turbulent flows. Phys. Rev. E 97, 053101.

Debue, P., Valori, V., Cuvier, C., Daviaud, F., Foucaut, J.-M., Laval, J.-P., Wiertel-Gasquet, C., PAdilla, V. \& Dubrulle, B. 2020 Three-dimensional analysis of precursors to non-viscous dissipation in an experimental turbulent flow. J. Fluid Mech. (in press). 


\section{On the nature of intermittency in a von Kármán flow}

Dubrulle, B. 1994 Intermittency in fully developed turbulence: log-Poisson statistics and generalized scale covariance. Phys. Rev. Lett. 73, 959-962.

Dubrulle, B. 2019 Beyond Kolmogorov cascades. J. Fluid Mech. 867, P1.

FARGE, M. 1992 Wavelet transforms and their applications to turbulence. Annu. Rev. Fluid Mech. 24 (1), 395-458.

FRISCH, U. \& PARISI, G. 1985 On the singularity structure of fully developed turbulence. In Turbulence and Predictability in Geophysical Fluid Dynamics and Climate Dynamics (ed. M. Gil, R. Benzi \& G. Parisi), pp. 84-88. Elsevier.

FrisCH, U. \& VERGASSOLA, M. 1991 A prediction of the multifractal model: the intermediate dissipation range. Europhys. Lett. 14 (5), 439-444.

Geneste, D., Faller, H., Nguyen, F., Shukla, V., Laval, J.-P., Daviaud, F., Saw, E.-W. \& Dubrulle, B. 2019 About universality and thermodynamics of turbulence. Entropy 21 (3), 326.

Kestener, P. \& ARneodo, A. 2004 Generalizing the wavelet-based multifractal formalism to vector-valued random fields: application to turbulent velocity and vorticity 3D numerical data. Phys. Rev. Lett. 93 (4), 044501.

Kolmogorov, A. 1941 The local structure of turbulence in incompressible viscous fluid for very large Reynolds' numbers. Akad. Nauk SSSR Dokl. 30, 301-305.

Kolmogorov, A.N. 1962 A refinement of previous hypotheses concerning the local structure of turbulence in a viscous incompressible fluid at high Reynolds number. J. Fluid Mech. 13 (1), 82-85.

Kraichnan, R.H. 1975 Remarks on turbulence theory. Adv. Maths 16 (3), 305-331.

KuzZay, D., Faranda, D. \& Dubrulle, B. 2015 Global vs local energy dissipation: the energy cycle of the turbulent von Kármán flow. Phys. Fluids 27, 075105.

LuO, G. \& Hou, T.Y. 2014 Potentially singular solutions of the 3D axisymmetric Euler equations. Proc. Natl Acad. Sci. 111 (36), 12968-12973.

Mailybaev, A.A. 2015 Continuous representation for shell models of turbulence. Nonlinearity 28 (7), 2497-2514.

MARIE, L. \& DAVIAUD, F. 2004 Experimental measurement of the scale-by-scale momentum transport budget in a turbulent shear flow. Phys. Fluids 16 (2), 457-461.

Meneveau, C. 1991 Analysis of turbulence in the orthonormal wavelet representation. J. Fluid Mech. 232, 469-520.

Moffatт, H.K. 2002 G.K. Batchelor and the homogenization of turbulence. Annu. Rev. Fluid Mech. 34 (1), 19-35.

Moisy, F. \& JiMÉnEZ, J. 2004 Geometry and clustering of intense structures in isotropic turbulence. J. Fluid Mech. 513, 111-133.

MuZY, J.F., BACRY, E. \& ARneOdo, A. 1991 Wavelets and multifractal formalism for singular signals: application to turbulence data. Phys. Rev. Lett. 67 (25), 3515.

Nguyen, F., Laval, J.-P., Kestener, P., Cheskidov, A., Shvydkoy, R. \& Dubrulle, B. 2019 Local estimates of Hölder exponents in turbulent vector fields. Phys. Rev. E 99 (5), 053114.

Nore, C., Castanon Quiroz, D., Cappanera, L. \& Guermond, J.-L. 2018 Numerical simulation of the von Kármán sodium dynamo experiment. J. Fluid Mech. 854, 164-195.

Ostovan, Y., Cuvier, Ch., Debue, P., Valori, V., Cheminet, A., Foucaut, J.-M., Laval, J.-Ph., Wiertel-Gasquet, C., Padilla, V. \& Dubrulle, B. 2019 4D particle tracking velocimetry measurements in a von Kármán turbulence experiment. In Proceedings of the 13th International Symposium on Particle Image Velocimetry (ed. C.J. Kähler, R. Hain, S. Scharnowski \& T. Fuchs).

Ouellette, N.T., Xu, H., Bourgoin, M. \& Bodenschatz, E. 2006 Small-scale anisotropy in Lagrangian turbulence. New J. Phys. 8 (6), 102-102.

PARET, J. \& TABEling, P. 1998 Intermittency in the two-dimensional inverse cascade of energy: experimental observations. Phys. Fluids 10 (12), 3126-3136.

Ravelet, F., Chiffaudel, A. \& Daviaud, F. 2008 Supercritical transition to turbulence in an inertially driven von Kármán closed flow. J. Fluid Mech. 601, 339-364.

Saw, E.-W., Debue, P., Kuzzay, D., Daviaud, F. \& Dubrulle, B. 2018 On the universality of anomalous scaling exponents of structure functions in turbulent flows. J. Fluid Mech. 837, 657-669.

Saw, E.-W., Kuzzay, D., Faranda, D., Guittonneau, A., Daviaud, F., Wiertel-Gasquet, C., Padilla, V. \& Dubrulle, B. 2016 Experimental characterization of extreme events of inertial dissipation in a turbulent swirling flow. Nat. Commun. 7, 12466.

SCHNEIDER, K. \& VASILYEV, O.V. 2010 Wavelet methods in computational fluid dynamics. Annu. Rev. Fluid Mech. 42 (1), 473-503.

Vincent, A. \& Meneguzzi, M. 1994 The dynamics of vorticity tubes in homogeneous turbulence. J. Fluid Mech. 258, 245-254. 